\title{
Detection and Analysis of Regional Trends of Klebsiella pneumonia Causing Liver Abscess
}

\author{
Yunfang Sun ${ }^{1,2}$, Hua $\mathbf{W u}^{1,3}$ and Dingxia Shen ${ }^{1^{*}}$ \\ ${ }^{1}$ Department of Microbiology, Chinese General Hospital of People's Liberation Army, Beijing 100853, China \\ ${ }^{2}$ Department of Microbiology, Shandong Medical College, Linyi, 276000, China \\ ${ }^{3}$ Department of Microbiology, Hainan Provincial People's Hospital, Haikou, 570311, China
}

*Corresponding author: Dingxia Shen, Department of Microbiology, Chinese General, Hospital of People's Liberation Army, Beijing 100853, China, Tel: +15866930039; E-mail: syfzlt@163.com

Received date: May 13, 2015; Accepted date: July 13, 2015; Published date: July 20, 2015

Copyright: ( 2015 Sun Y, et al. This is an open-access article distributed under the terms of the Creative Commons Attribution License, which permits unrestricted use, distribution, and reproduction in any medium, provided the original author and source are credited.

\begin{abstract}
Background: Hypervirulent variant of Klebsiella pneumoniae is the major pathogen causing liver abscess.

Methods: A retrospective study was conducted in 240 patients with cultures positive for $K$. pneumoniae hospitalized in the Chinese PLA General Hospital from May 2013 to August 2014. The clinical and molecular data of the hypervirulent $K$. pneumoniae (hvKP) causing liver abscess were analyzed.

Results: Among 240 strains of $K$. pneumoniae, hvKP accounted for 42.5\% (102/240), hvKP causing liver abscess were 37 strains, accounting for $36.3 \%$ (37/102), patients with diabetes were $11(11 / 37,29.7 \%), 13$ $(13 / 37,35.1 \%)$ patients were diagnosed as fever of unknown origin at first, $7(7 / 37,18.9 \%)$ patients with tumor, the rest $6(6 / 37,16.2 \%)$ patients with postoperative infection or other site infection. Univariate analysis revealed the following risk factors for hvKP causing liver abscess: string test (odds ratio (OR), 11.306 [95\% confidence interval (Cl), 3.579-35.711]), serotype K1 (OR, 3.109 [95\% Cl, 1.338-7.222]) and fever of unknown origin (OR, 6.921 [95\% $\mathrm{Cl}, 2.503-19.136])$. The results detected by multiplex PCR were consistent with single PCR. 102 strains of hvKP were tested the sensitivity to $14-19$ drug, 37 strains caused liver abscess were not found with ESBL.
\end{abstract}

Conclusions: The detection of string test combined with rmpA and aerobactin can better identify hvKP. Patients with liver abscess generally were diabetes, but some patients didn't have other disease. There is not much difference about the chance to be attacked by hvKP among male and female. ESBL was not detected among the hvKP causing liver abscess. Multiplex PCR assay could detect hvKP quickly.

Keywords: K. pneumoniae, Hypervirulent; Epidemiology; Detection; Clinical features

\section{Introduction}

In recent years, $K$. pneumoniae has become the second largest bacteria after the $E$. coli in China causing community-acquired infections and nosocomial infection [1]. The $K$. pneumoniae strains commonly recognized by clinicians and microbiologists are termed classic $K$. pneumoniae (cKP). Such strains are notorious for their capacity to cause nosocomial infections and acquire antimicrobial resistance [2,3]. A new variant of $K$. pneumoniae, designated as hypervirulent $K$. pneumoniae (hvKP), was first described in 1986 by a group of Taiwanese doctors reporting a clinical syndrome of community-acquired $K$. pneumoniae infections [4-6]. Compared with ordinary pneumonia Klebsiella, hvKP is able to effectively produce capsule, the formation of high mucoid phenotype with high virulence factors, the hvKP strains exhibit a striking capacity to cause serious infections such as pneumonia, liver abscess, encephalitis, endophthalmitis, biliary tract inflammation [7]. hvKP colonies grown on agar exhibit hypermucoviscosity. This phenotype has been used as a standard laboratory test to distinguish hvKP from cKP and is defined as a positive "string test" [8]. Liver abscess is a serious disease of digestive system, single or multiple. From the beginning of 1990s, hvKP gradually become the main pathogenic bacteria of liver abscess from Escherichia coli, streptococci and enterococci. It is easy to cause infection of other parts by transfer and the time of therapy is long, sometimes leads to recurrence, sometimes combined with other serious disease even to death [9]. An increasing number of cases of liver abscess due to hvKP are present in various Western countries and an awareness of this entity, which may be complicated by severe sepsis or metastatic infection is needed. Recently Romania found liver abscess associated with severe myopathy caused by $K$. pneumoniae serotype $\mathrm{K} 1$ [3]. In Brazil, there was a case of $\mathrm{K} 1$ type causing liver abscess and finally to death [5]. The traits that enhance its virulence when compared with cKP are the ability to more efficiently acquire iron and perhaps an increase in capsule production, which confers the hypermucoviscous phenotype. An objective diagnostic test suitable for routine use in the clinical microbiology laboratory is needed. At present, hvKP is becoming more and more popular in China, there are a lot of reports about the isolation and identification, drug resistance [6]. But how to define hvKP is still lack of unified standards [9]. Most of the hvKP strains identified to date are susceptible to antimicrobials with the exception of ampicillin [6]. However, the enhanced hvKP strains now have acquired antimicrobial resistance in some hospitals in China [8]. Combined with the increased risk to susceptible 
populations, these issues have attracted calls for preemptive intervention to mitigate the possibility of the globally damaging effects of hvKP infections. We conducted a retrospective analysis of 240 patients with positive cultures for $K$. pneumoniae hospitalized in General Hospital of PLA from May 2013 to August 2014, detected the mucoid phenotype, the capsule serotypes and the main virulence genes by multiplex PCRanalysed the clinical characteristics with disease, hope it can provide reference to laboratory diagnosis and treatment of hvKP.

\section{Materials and Methods}

\section{Patient Information}

A retrospective study was conducted on 240 consecutive $K$. pneumoniae culture-positive patients hospitalized at General Hospital of PLA from May 2013 to August 2014. Clinical and laboratory data were gathered and analysis was made among patients from which hvKP strains were isolated.

The protocol for this study was approved by General Hospital of PLA Ethics Committee, and the Guidelines for Human Experimentation (PRC) were followed throughout. All patients gave written informed consent upon admission for their information to be stored and used for research.

\section{Clinical isolates of $K$. pneumonia}

$240 \mathrm{KP}$ strains were from blood infection, respiratory tract infection, urinary tract infection, liver abscess patients, isolated from blood, sputum, bronchoalveolar lavage fluid, urine, liver abscess and other abscess puncture fluid, bile, sterile site of puncture fluid (pleural effusion and ascites and cerebral spinal fluid and synovial fluid), drainage liquid, central catheter. All of the strains were identified as $K$. pneumonia by using an automated bacterial identification system (VITEK $^{\circ}$ 2, bioMe rieux, Hazelwood, MI, USA), some bacteria were confirmed through mass spectrometer.

\section{Clinical Microbiologic Characterization of the $K$. pneumoniae Strains}

All $K$. pneumoniae isolates were frozen and stored at $-80^{\circ} \mathrm{C}$. Susceptibility testing (Phoenix 100 automated microbiology system, BD, Franklin Lakes, New Jersey) to Amikacin, Amoxicillinclavulanate, Ampicillin, Ampicillin-sulbactam, Aztreonam, Ceftizoxime, Cefepime, Cefotaxime, Ceftazidime, Ciprofloxacin, Centamicin, Levofloxacin, Piperacillin, Tetracycline, Trimethoprimsulfamethoxazole, Chloramphenicol, Imipenem, Meropenem, and Piperacillin-tazobactam was conducted for every strain. Extended spectrum $\beta$-lactamase (ESBL) production was also determined using the Phoenix 100 system. A string test, arobactin and $\mathrm{rmpA}$ were performed to distinguish hvKP from cKP. K. pneumoniae strains with a positive string test or arobactin or rmpA were designated as hvKP. Arobactin and rmpA are virulence genes of $K$. pneumoniae. Khe gene was detected to identify the common attributes of $K$. pneumoniae, K1, K2, K5, K16, K20, K54 and K57 were detected to establish the capsular serotypes.

\section{String test}

A string test was performed to distinguish hvKP from cKP. A positive string test was defined as the formation of a mucoviscous string of $>5 \mathrm{~mm}$, when using a bacteriology inoculation loop to stretch a colony grown overnight on an agar plate at $37^{\circ} \mathrm{C} . K$. pneumoniae strains with a positive string test were designated as hvKP.

\section{Polymerase chain reaction-mediated detection of $\mathrm{RmpA}$, arobactin, and capsular serotype-specific genes}

Genomic DNA was extracted from all $K$. pneumoniae strains (Thermal cracking method) and the rmpA, arobactin, and serotypespecific genes for the K1, K2, K5, K16, K20, K54, and K57 capsular serotypes were amplified by polymerase chain reaction (ABI Veriti Thermal Cycler, Applied Biosystems Asia Pte Ltd., Singapore) Capsule type genes and virulence genes amplification primers synthesized by Shanghai shenggong. The total volume of the reaction is $25 \mu \mathrm{l}$ and contains 10 kinds of primers. Ten kinds of primers were K1, K2, K54, $\mathrm{K} 57, \mathrm{rmpA}$. In another group ten kinds of primers were K5, Khe, arobactin, K16, K20. $0.5 \mu \mathrm{l}$ dNTPS (each dNTP final concentration of $0.2 \mathrm{mmol} / \mathrm{l})$. Primers F $(0.6 \mu \mathrm{mol} / \mathrm{l})$ and primer $\mathrm{R}(0.6 \mu \mathrm{mol} / \mathrm{l}) 1 \mu \mathrm{l}$, Taq enzyme is $0.5 \mu \mathrm{l}, 2 \mu \mathrm{l}$ templates. The primers used are listed in Table 1.

\begin{tabular}{|c|c|}
\hline Primer Name & Sequence \\
\hline \multicolumn{2}{|l|}{ khe } \\
\hline Forward & 5-TGATTGCATTCGCCACTGG-3 \\
\hline Reverse & 5-GGTCAACCCAACGATCCTG-3 \\
\hline \multicolumn{2}{|l|}{ arobactin } \\
\hline Forward & 5-GCATAGGCGGATACGAACAT-3 \\
\hline Reverse & 5-CACAGGGCAATTGCTTACCT-3 \\
\hline \multicolumn{2}{|l|}{ rmpA } \\
\hline Forward & 5-ACTGGGCTACCTCTGCTTCA-3 \\
\hline Reverse & 5-CTTGCATGAGCCATCTTTCA-3 \\
\hline \multicolumn{2}{|l|}{ K1 } \\
\hline Forward & 5-GTAGGTATTGCAAGCCATGC-3 \\
\hline Reverse & 5-GCCCAGGTTAATGAATCCGT-3 \\
\hline \multicolumn{2}{|l|}{ K2 } \\
\hline Forward & 5-GGAGCCATTTGAATTCGGTG-3 \\
\hline Reverse & 5-TCCСTAGCACTGGCTTAAGT-3 \\
\hline \multicolumn{2}{|l|}{ K5 } \\
\hline Forward & 5-GCCACCTCTAAGCATATAGC-3 \\
\hline Reverse & 5-CGCACCAGTAATTCCAACAG-3 \\
\hline \multicolumn{2}{|l|}{ K16 } \\
\hline Forward & 5-GTGCTTAACGGAGAACTGAAC-3 \\
\hline Reverse & 5-ССTCACCTGGAAGAAGTGTA-3 \\
\hline \multicolumn{2}{|l|}{ K20 } \\
\hline Forward & 5-CCGATTCGGTCAACTAGCTT-3 \\
\hline Reverse & 5-GCACCTCTATGAACTTTCAG-3 \\
\hline
\end{tabular}


Page 3 of 5

\begin{tabular}{|ll|c|}
\hline K54 & \multicolumn{2}{|c|}{} \\
\hline & Forward & 5-CATTAGCTCAGTGGTTGGCT-3 \\
\hline & Reverse & 5-GCTTGACAAACACCATAGCAG-3 \\
\hline $\mathbf{K 5 7}$ & & \\
\hline & Forward & 5-CGACAAATCTCTCCTGACGA-3 \\
\hline & Reverse & 5-CGCGACAAACATAACACTCG-3 \\
\hline
\end{tabular}

Table 1: Primers.

\section{Statistical analysis}

SPSS software (version 15.0) was used for data analysis. Logistic regression was used to analyze risk factors for hvKP. P values of $<0.05$ were considered statistically significant.

\section{Results}

\section{Patient characteristics}

From May 2013 to August 2014, a total of 240 patients admitted to General Hospital of PLA were identified as having cultures positive for $K$. pneumoniae at 1 or more sites. Isolates of hvKP and cKP were obtained from $102(43.3 \%)$ and $138(57.5 \%)$ of patients, respectively. The strains were isolated from blood (cKP 71.7\%, hvKP 28.3\%), urine (cKP 86.2\%, hvKP 13.8\%), sputum (cKP 53.7\%, hvKP46.3\%), ascites (cKP 50\%, hvKP 50\%), bile (cKP63.2\%, hvKP36.8\%), and abscess fluid (cKP39.1\%, hvKP60.9\%). A significantly higher number of patients with hvKP in abscess fluid were detected. No significant differences were detected in the other sample types.

37 cases of liver abscess were caused by hvKP. 43.3\% (102/240) of $K$. pneumoniae were hvKP, 37 strains caused liver abscess, accounting for
$35.6 \%$ (37/102). CKP accounted for 57.5\% (138/240), only 1 strain caused liver abscess, accounting for $0.07 \%$ (1/138). Among liver abscess patients caused by 37 hvKP strains, 11 patients were diabetic, 13 patients were diagnosed as fever of unknown origin at first, 7 were tumor patients, and 4 patients were with postoperative infection or other site infection.

\section{Genetic characteristics of hvKP}

String test was positive in 79 strains hvKP, accounting for $77 \%$ (79/102); the rmpA positive strains were 93, accounting for $91 \%$ (93/102); the aerobactin positive strains were 92 , accounting for $86 \%$ (88/102). 34 strains were K1, accounting for 33.3\% (34/102); type K2 29 strains, accounting for 28.4\%(29/102); type K5 9 strains, accounting for $8.8 \%$ (9/102); type K16 0 strains; 3 strains of K20, accounting for $2.9 \%$ (3/102); type K54 8 strains, accounting for 7.8\% (8/102); 6 strains of type K57, accounting for $5.9 \%(6 / 102)$; the remaining 6 strains with virulence factors but were not identified as related type strains, accounting for $5.9 \%(6 / 102)$. K1, K2, K5, K54 were positively correlated to hvKP. All K. pneumoniae were positive in Khe test. Nontoxic factor of K5 were $40 \%(6 / 15)$, K57 strain without virulent factor were $14.3 \%(1 / 7), 6.5 \%(2 / 31)$ strains of $\mathrm{K} 2$ have no virulence factors, $12.7 \%(13 / 102)$ with virulence factor but the type were not identified as related type. In the $37 \mathrm{hvKP}$ strains caused liver abscess, string test positive strains were 31 , accounted for $83.8 \%$ (31/37); the rmpA positive strains of 35 , accounting for $94.6 \%$ (35/37); the aerobactin positive strains of 32 , accounting for $86.5 \%$ (32/37); 21 strains of type K1, accounting for $56.8 \%$ (21/37); type K2 10 strains, accounting for $29.7 \%$ (11/37); type K5 4 strains, accounting for $10.8 \%$ (4/37); type K54 1 strains, accounting for $2.7 \%$ (1/37), 1 strain without type.

Multiple PCR detection results were consistent with single PCR (Table 2).

\begin{tabular}{|c|c|c|c|c|c|}
\hline Characteristic & String test, No. & rmpA, No. & abc, No. & No virulence, No. & Liver abscess, No. \\
\hline \multicolumn{6}{|l|}{ K serotype } \\
\hline K1 & $30 / 34(88 \%)$ & $34 / 34(100 \%)$ & $32 / 34(94 \%)$ & 0 & $21 / 37(56.8 \%)$ \\
\hline $\mathrm{K} 2$ & 25/31 (81\%) & $25 / 31(81 \%)$ & $28 / 31(90 \%)$ & $2 / 31$ & $11 / 37(29.7 \%)$ \\
\hline K5 & $7 / 15(47 \%)$ & $8 / 15(53 \%)$ & $6 / 15(40 \%)$ & $6 / 51$ & $4 / 37(10.8 \%)$ \\
\hline $\mathrm{K} 20$ & $2 / 3(67 \%)$ & $3 / 3(100 \%)$ & $3 / 3(100 \%)$ & 0 & 0 \\
\hline K54 & $3 / 8(38 \%)$ & $6 / 8(75 \%)$ & $8 / 8(100 \%)$ & 0 & $1 / 37(2.7 \%)$ \\
\hline K57 & $6 / 7(86 \%)$ & $6 / 7(86 \%)$ & $6 / 7(86 \%)$ & 1 & 0 \\
\hline K-nontypable & $6 / 13(46 \%)$ & $13 / 13(100 \%)$ & $5 / 13(38 \%)$ & 0 & $1 / 37(2.7 \%)$ \\
\hline Total & $79 / 102(77 \%)$ & $93 / 102(91 \%)$ & $88 / 102(86 \%)$ & 9/102 (9\%) & \\
\hline
\end{tabular}

Table 2: Characteristics of $K$. pneumoniae strains.

\section{Risk factors}

Univariate analysis showed that $\mathrm{K} 1$ (odds ratio $(\mathrm{OR})=3.109$ ), positive string test $(\mathrm{OR}=11.306)$ and fever of unknown origin $(\mathrm{OR}=6.921)$ were statistically significant risk factors for liver abscess. $\operatorname{RmpA}(\mathrm{OR}=0.571), \quad \mathrm{K} 2(\mathrm{OR}=0.821)$, aerobactin $(\mathrm{OR}=3.632)$, diabetes $(\mathrm{OR}=2.263), \mathrm{K} 5(\mathrm{OR}=1.041)$ appeared to have not much difference between hvKP caused liver abscess and hvKP caused other diseases (Table 3). 


\section{Antimicrobial resistance among hvKP isolates}

All hvKP strains were resistant to Ampicillin, which is consistent with previous studies $[9,10]$. However, resistance to all the tested antimicrobials, except Carbapenems and Amikacin, was observed in a proportion of hvKP strains, none of which expressed ESBL in the 37 hvKP causing liver abscess.

\begin{tabular}{|c|c|c|}
\hline Risk Factor & \multicolumn{2}{|c|}{ Univariate Analysis } \\
\hline & OR (95\% Cl) & P Value \\
\hline rmpA & $0.571(0.035-9.415)$ & 0.695 \\
\hline Aerobactin & $3.632(0.759-13.787)$ & 0.106 \\
\hline Capsule antigen K1 & $3.109(1.338-7.222)$ & 0.008 \\
\hline Capsule antigen K2 & $0.821(0.325-2.077)$ & 0.678 \\
\hline Capsule antigen K5 & $1.041(0.234-4.630)$ & 0.958 \\
\hline Fever of unknown origin & $6.921(2.503-19.136)$ & 0 \\
\hline Diabetes mellitus & $2.263(0.823-6.224)$ & 0.113 \\
\hline Positive string test & $11.306(3.579-35.711)$ & 0 \\
\hline
\end{tabular}

Table 3: Risk Factors for hvKP causing liver abscess vs hvKP causing other disease.

\section{Discussion}

It is well known that $K$. pneumoniae is a common pathogen responsible for pneumonia as well as blood and urinary tract infections, easy to form resistance to antibiotics [11-13]. Recently, lifethreatening liver abscess caused by $K$. pneumoniae has been paid more and more attention, the therapy time is longer, sometime easy to attack again, significant morbidity and mortality occurs [14]. Liver abscess caused by $K$. pneumonia this mostly were highly mucoid phenotype in biological classification, has some unique capsular types and virulence factors, known as hvKP [8]. Usually the hvKP identification is based on the string test, the identification of hvKP need a certain standard [9]. More experiments are needed to establish whether a virulence factor or property present in both cKP and hvKP is equally important for their pathogenesis or whether it accounts for the increased virulence of hvKP strains compared with cKP strains. Standard need to be make clear to make a clinical laboratory diagnosis as soon as possible to help doctors diagnose as quickly as they can so that they can make correct treatment and prevent metastasis and drug resistance. This retrospective study was conducted in $240 \mathrm{~K}$. pneumoniae culture-positive patients hospitalized during the period from May 2013 to August 2014 in General Hospital of PLA. Anyone positive of rmpA, arobactin and string test can be diagnosed as hvKP. The clinical and biological characteristics analysis indicated that this strain identification method can be a better method to identify hvKP. No ESBL was found in the 37 hvKP which caused liver abscess. On the other hand, the study showed that multiplex PCR to detect hvKP by virulent type and capsule type can be more quickly to make the laboratory identification of clinical specimens than traditional methods.

HvKP detection in pus specimens is higher than other specimens. Other samples for hvKP detection from high to low in turn are ascites, sputum, blood, urine, bile. Some studies have shown that infections caused by hvKP are more invasive and serious in healthy young people [10]. This study found 37 cases of liver abscess patients aged for a minimum of 20 years, the oldest was 79 years, the average age is 55 years; 18 of 37 cases were female, 19 were male, the data showed age and gender have less correlation with liver abscess. 13 of 37 patients with liver abscess at first diagnosed as unknown origin fever, 11 were diabetes, and 7 were tumor patients and 4 patients with postoperative infection or other site infection. The 13 patients with no other concurrent disease diagnosed as unknown origin fever finally was identified as liver abscess. As a result, liver abscess can be independently caused by hvKP and indicated that patients with diabetes mellitus is a relatively high risk factor, this is consistent with the majority of research $[13,15]$.

Genes that encode a number of virulence factors, including those that are responsible for the hypermucoviscous phenotype (rmpA) and iron-acquisition factors (aerobactin). Among the $37 \mathrm{hvKP}$ caused liver abscess, positive string test were 31 , accounted for $83.8 \%(31 / 37)$; rmpA positive strains accounting for $94.6 \%$ (35/37); aerobactin positive strains accounting for $86.5 \%(32 / 37)$. It is clear that not all hvKP are hypermucoviscous. The experiments showed that the combination of rmpA, arobactin, string test can be a better method to identify hvKP infection compared to just depend on string test. Once some specimens were laboratory diagnosed as hvKP, clinicians could make timely treatment to prevent further spread of infection.

37 strains of $K$. pneumoniae caused liver abscess were all hvKP, 21 were $\mathrm{K} 1$, accounting for $56.8 \%$ (21/37); 10 were K2, accounting for $29.7 \%$ (11/37); 4 were K5, accounting for $10.8 \%(4 / 37)$; 1 is K54, accounting for $2.7 \%(1 / 37)$ and 1 was not the type to be detected. 35 strains expressed rmpA, accounting for $94.6 \%$ (35/37), among the 37 strains of hvKP, rmpA all were expressed in $21 \mathrm{~K} 1$. These data are consistent with previous reports [15-17]. Single factor regression analysis showed that $\mathrm{K} 1$, string test positive and unexplained fever is the risk factors to liver abscess compared to hvKP causing other disease.

Multiple PCR detection results were consistent with single PCR. Then the detection of hvKP can be quicker by using multiple PCR and can be used as a rapid method to identify hvKP in clinical laboratory.

Compounding an already challenging clinical situation is the recent propensity for $\mathrm{cKP}$ to become multi-, extreme or pan-drug-resistant, including the acquisition of extended-spectrum $\beta$-lactamases and carbapenemases, such as the recently described NDM-1[18]. To date, most strains of hvKP have been very susceptible to antimicrobials except ampicillin [19]. Nonetheless, some cases of infection due to MDR-hvKP have already been described $[8,20]$. The confluence of hypervirulence and extreme or pan-drug resistance in hvKP has the potential to create a "post-antibiotic" scenario. In this study, we compared the characteristics of drug resistance of isolated hvKP. In the present study of the 14-19 drug resistance tests, the acquisition of antimicrobial resistance to some drugs was existed, but there was no ESBL resistant strain existed in the $37 \mathrm{hvKP}$ causing liver abscess.

In conclusion, the research of hvKP is susceptible to most antibiotics, the string test and multiplex PCR to virulence genes, capsular phenotype can quickly make the rapid identification of hcKP. This will help to make clinical diagnosis quickly and avoid the further development of the disease and reduce drug-resistant strains. 


\section{References}

1. Jinyu $\mathrm{He}$ (2012) Study on the distribution pattern of the serum and virulence genes of K.pneumoniae. Chinese outstanding master's degree thesis database.

2. Zhang Y, Yang J, Ye L, Luo Y, Wang W, et al. (2012) Characterization of clinical multidrug-resistant Escherichia coli and Klebsiella pneumoniae isolates, 2007-2009, China. Microb Drug Resist 18: 465-470.

3. Popescu GA, Tanase D, Petrescu AM, Florea D (2014) Liver abscess associated with severe myopathy caused by K. pneumoniae serotype K1 in Romania. J Infect Dev Ctries 8: 1491-1493.

4. Li B, Zhao Y, Liu C, Chen Z, Zhou D (2014) Molecular pathogenesis of Klebsiella pneumoniae. Future Microbiol 9: 1071-1081.

5. Coutinho RL, Visconde MF, Descio FJ, Nicoletti AG, Pinto FC, et al. (2014) Community-acquired invasive liver abscess syndrome caused by a K1 serotype Klebsiella pneumoniae isolate in Brazil: a case report of hypervirulent ST23. Mem Inst Oswaldo Cruz 109: 970-971.

6. Sobirk SK, Struve C, Jacobsson SG (2010) Primary Klebsiella pneumoniae Liver Abscess with Metastatic Spread to Lung and Eye, a North-European Case Report of an Emerging Syndrome. Open Microbiol J 4: 5-7.

7. Chan DS, Archuleta S, Llorin RM, Lye DC, Fisher D (2013) Standardized outpatient management of Klebsiella pneumoniae liver abscesses. Int J Infect Dis 17: e185-188.

8. Li W, Sun G, Yu Y, Li N, Chen M, et al. (2014) Increasing occurrence of antimicrobial-resistant hypervirulent (hypermucoviscous) Klebsiella pneumoniae isolates in China. Clin Infect Dis 58: 225-232.

9. Shon AS, Bajwa RP, Russo TA (2013) Hypervirulent (hypermucoviscous) Klebsiella pneumoniae: a new and dangerous breed. Virulence 4: 107-118.

10. Ma LC, Fang CT, Lee CZ, Shun CT, Wang JT (2005) Genomic heterogeneity in Klebsiella pneumoniae strains is associated with primary pyogenic liver abscess and metastatic infection. J Infect Dis 192: 117-128.
11. Turton JF, Perry C, Elgohari S, Hampton CV (2010) PCR characterization and typing of Klebsiella pneumoniae using capsular type-specific, variable number tandem repeat and virulence gene targets. J Med Microbiol 59: 541-547.

12. Wang JH, Liu YC, Lee SS, Yen MY, Chen YS, et al. (1998) Primary liver abscess due to Klebsiella pneumoniae in Taiwan. Clin Infect Dis 26: 1434-1438.

13. Keynan Y, Karlowsky JA, Walus T, Rubinstein E (2007) Pyogenic liver abscess caused by hypermucoviscous Klebsiella pneumoniae. Scand J Infect Dis 39: 828-830.

14. Pomakova DK, Hsiao CB, Beanan JM, Olson R, Macdonald U, et al. (2012) Clinical and phenotypic differences between classic and hypervirulent Klebsiella pneumonia: an emerging and under-recognized pathogenic variant. Eur J Clin Microbiol Infect Dis 31: 981-989.

15. Snitkin ES, Zelazny AM, Thomas PJ, Stock F; NISC Comparative Sequencing Program Group, Henderson DK, et al. (2012) Tracking a hospital outbreak of carbapenem-resistant Klebsiella pneumoniae with whole-genome sequencing. Sci Transl Med 4: 148ra116.

16. Enani MA, El-Khizzi NA (2012) Community acquired K. pneumoniae, K1 serotype. Invasive liver abscess with bacteremia and endophthalmitis. Saudi Med J 33: 782-786

17. Turton JF, Englender H, Gabriel SN, Turton SE, Kaufmann ME, et al. (2007) Genetically similar isolates of Klebsiella pneumoniae serotype K1 causing liver abscesses in three continents. J Med Microbiol 56: 593-597.

18. Moellering RC Jr (2010) NDM-1-a cause for worldwide concern. N Engl J Med 363: 2377-2379.

19. Lin YT, Jeng YY, Chen TL, Fung CP (2010) Bacteremic communityacquired pneumonia due to Klebsiella pneumoniae: clinical and microbiological characteristics in Taiwan, 2001-2008. BMC Infect Dis 10: 307.

20. Cheng NC, Yu YC, Tai HC, Hsueh PR, Chang SC, et al. (2012) Recent trend of necrotizing fasciitis in Taiwan: focus on monomicrobial Klebsiella pneumoniae necrotizing fasciitis. Clin Infect Dis 55: 930-939. 